\title{
AUTHOR INDEX Volume 3
}

Arbieto, A. \& Bochi, J., $L^{p_{-}}$ generic cocycles have onepoint Lyapunov spectrum

Arbieto, A. \& Bochi, J., Corrigendum: $\quad L^{p}$-generic cocycles have one-point Lyapunov spectrum

Bahsoun, W., Góra, P. \& Boyarsky, A., Stochastic perturbations of position dependent random maps

Belinskiy, B.P. \& Caithamer, P., Stability of dynamical systems with a multiplicative white noise

Bochi, J., see Arbieto

Bochi, J., see Arbieto

Boyarsky, A., see Bahsoun

Burton, R.M., Dehling, H.G. \& Rösler, U., A law of large numbers for rescaled random difference equations

Caithamer, P., see Belinskiy

Caraballo, T., Garrido-Atienza, M.J. \& Real, J., The exponential behaviour of nonlinear stochastic functional equations of second order in time

Carmona, S.C. \& Freidlin, M.I., On logarithmic asymptotics of stochastic resonance frequencies

3 (2003) 73

3 (2003) 419

3 (2003) 545

3 (2003) 187

3 (2003) 73

3 (2003) 419

3 (2003) 545

3 (2003) 409

3 (2003) 187

3 (2003) 169

3 (2003) 55

3 (2003) 409

Dehling, H.G., see Burton

Duc, L.H., On the absolute regularity of linear random dynamical systems

3 (2003) 453

Erraoui, M., Nualart, D. \& Ouknine, Y., Hyperbolic stochastic partial differential equations with additive fractional Brownian sheet

Freidlin, M.I., see Carmona

3 (2003) 121

3 (2003) 55

Freidlin, M.I. \& Wentzell, A.D., Averaging principle for stochastic perturbations of multifrequency systems

Garrido-Atienza, M.J. \& Real, J., Existence and uniqueness of solutions for delay stochastic evolution equations of second order in time

3 (2003) 393

3 (2003) 141
Garrido-Atienza, M.J., see Caraballo

3 (2003) 169

Góra, P., see Bahsoun

3 (2003) 545

Guillotin-Plantard, N. \& Schneider, D., Limit theorems for sampled dynamical systems Imai, A., Kawasaki, Y. \& Sato, H., Martin metrics on the Sierpiński gasket

Imkeller, P. \& Kloeden, P., On the computation of invariant measures in random dynamical systems

3 (2003) 477

3 (2003) 267

Kawasaki, Y., see Imai

Khasminskii, R. \& Klebaner, F., A note on averaging and homogenization

Kifer, Y., $L^{2}$ diffusion approximation for slow motion in averaging

Klebaner, F., see Khasminskii

Kloeden, P.E., Pullback attractors of nonautonomous semidynamical systems

Kloeden, P., see Imkeller

Koch, S., Furstenberg-type formulas over shift spaces

Lamberton, D. \& Pagès, G., Recursive computation of the invariant distribution of a diffusion: The case of a weakly mean reverting drift

3 (2003) 435

Lawrance, A.J. \& Wolff, R.C., Binary time series generated by chaotic logistic maps

Marín-Rubio, P. \& Robinson, J.C., Attractors for the stochastic 3D Navier-Stokes equations

Nakada, H. \& Natsui, R., Some strong mixing properties of a sequence of random variables arising from $\alpha$-continued fractions

Natsui, R., see Nakada

Nualart, D., see Erraoui

O'Bryant, N.V., A noisy system with a flattened Hamiltonian and multiple time scales

Ouknine, Y., see Erraoui

Pagès, G., see Lamberton

3 (2003) 247

3 (2003) 267

3 (2003) 113

3 (2003) 213

3 (2003) 113

3 (2003) 101

3 (2003) 247

3 (2003) 499

3 (2003) 529

3 (2003) 279

3 (2003) 463

3 (2003) 463

3 (2003) 121

3 (2003) 1

3 (2003) 121

3 (2003) 435 
Real, J., see Caraballo

Robinson, J.C., see Marín-Rubio

Rösler, U., see Burton

Sato, H., see Imai

Schneider, D., see GuillotinPlantard

Sowers, R.B., Stochastic averaging near a homoclinic orbit with multiplicative noise

\author{
3 (2003) 169 \\ 3 (2003) 279 \\ 3 (2003) 409 \\ 3 (2003) 267 \\ 3 (2003) 477 \\ 3 (2003) 299
}

Wentzell, A.D., see Freidlin

Wolff, R.C., see Lawrance

Zverev, V.V., Noise transformation in nonlinear system with intensity dependent phase rotation

Zweimüler, R., Stable limits for probability preserving maps with indifferent fixed points
3 (2003) 421

3 (2003) 393

3 (2003) 529

3 (2003) 83 